ÉGYPTE monde arabe

\section{Égypte/Monde arabe}

12-13 | 1993

Une économie en transition

\title{
Les firmes égyptiennes de droit des affaires internationales
}

Jean-Claude Delaunay

\section{OpenEdition}

1 Journals

Édition électronique

URL : https://journals.openedition.org/ema/1250

DOI : 10.4000/ema. 1250

ISSN : 2090-7273

\section{Éditeur}

CEDEJ - Centre d'études et de documentation économiques juridiques et sociales

\section{Édition imprimée}

Date de publication : 31 mars 1993

Pagination : $73-100$

ISSN : 1110-5097

\section{Référence électronique}

Jean-Claude Delaunay, «Les firmes égyptiennes de droit des affaires internationales », Égypte/Monde arabe [En ligne], 12-13 | 1993, mis en ligne le 08 juillet 2008, consulté le 07 juillet 2022. URL : http:// journals.openedition.org/ema/1250; DOI : https://doi.org/10.4000/ema.1250

Ce document a été généré automatiquement le 7 juillet 2022.

Tous droits réservés 


\title{
Les firmes égyptiennes de droit des affaires internationales
}

\author{
Jean-Claude Delaunay
}

1 Au fur et à mesure que les pays - quel que soit leur niveau d'industrialisation prennent place dans le processus de mondialisation des économies, s'affirme pour eux le besoin d'une activité juridique de très haut niveau, notamment dans le domaine des affaires internationales. En effet, la multiplication des échanges de marchandises et de capitaux nécessite, pour chacun, l'intervention elle-même multipliée de ces intermédiaires particuliers que sont les juristes. Ce fait, aisément observable, est une des manifestations de la complexité des relations marchandes contemporaines, qui mettent en rapport, avec une fréquence croissante, des lieux éloignés les uns des autres, non seulement du point de vue géographique mais encore du point de vue sociologique - institutions, lois, niveaux de développement, cultures...

2 Les juristes spécialisés en droit des affaires internationales apparaissent, dès lors, comme des médiateurs de premier plan. Ils informent et conseillent les agents économiques, nationaux et étrangers qui, dans un sens ou dans l'autre, traversent la frontière. Ils accompagnent, à l'aide de contrats, les opérations commerciales et les investissements qui en résultent. Ils défendent et représentent les intérêts de leurs clients lorsque naissent les conflits.

3 L'importance de l'activité juridique pour le bon déroulement des affaires n'est guère sensible aux économistes français, qu'ils soient de tradition classique ou walrassienne. Habitués à raisonner sur des quantités sans tenir compte des institutions dans l'enveloppe desquelles elles fonctionnent réellement comme quantités économiques ${ }^{1}$, accoutumés à l'examen de systèmes clos supposés homogènes, ils ont, comme l'expliquait l'économiste américain John R. Commons au début des années 1930, traité des échanges et non des transactions ${ }^{2}$. Ils ont étudié le transfert des biens et des services sans considération du transfert correspondant des droits de propriété. La mondialisation des économies oblige, de nos jours, à redécouvrir l'importance de cette distinction. Car la réalité observable de la vie économique internationale est constituée de transactions et non d'échanges. Pour que les échanges aient lieu, il faut que les 
opérations parallèles portant sur le transfert des droits de propriété réussissent, alors que bien des ruptures peuvent se produire à ce niveau.

Dans la mesure où la mondialisation signifie l'existence d'une liaison étroite entre croissance interne et relations avec le reste du monde, les juristes impliqués dans le droit des affaires internationales occupent une position stratégique|pour le développement économique de chaque pays comme pour celui du commerce international. Tel est le contexte théorique ayant présidé à l'enquête que nous avons effectuée, en juillet 1991, auprès de 26 firmes juridiques égyptiennes ${ }^{3}$. C'est, en effet, ce type de firme, et non les départements juridiques internes aux entreprises qui, en Égypte, assurent aujourd'hui, de la façon la plus efficace et la plus créatrice, la fonction d'interface entre les opérateurs économiques nationaux et étrangers.

Alors que l'Égypte, sous la pression des organismes internationaux de crédit et de financement comme de celle de ses créanciers, s'engage depuis janvier 1991, plus qu'elle ne l'avait fait jusqu'alors, dans la voie de la libéralisation de son économie, il semblait important d'étudier les firmes juridiques de ce pays. Sans doute ne sont-elles que l'un des rouages de la société moderne. Elles n'en paraissent pas moins décisives pour le succès des réformes en cours. Les firmes juridiques de droit international forment objectivement aujourd'hui, en Égypte, une «industrie de pointe ». Elles sont tout aussi nécessaires au fonctionnement de ce pays que le sont les laboratoires de recherche, les banques ou les infrastructures de télécommunication.

6 Cependant, dans l'ambiance de notre époque, encore très marquée par la prédominance de la technique visible et de la production tangible, les firmes juridiques ne sont pas considérées comme des lieux stratégiques pour le développement. La société égyptienne (il en est également ainsi dans la société française, bien qu'à un moindre degré) renvoie à ses juristes une image négative d'eux-mêmes. Les juristes de droit international privé sont englobés dans cette perception. Ce sont, selon ces approches, tout au plus des « chirurgiens » ou des " pompiers », c'est-à-dire des personnes agissant sur les conséquences d'actes ou de situations passés. On ne considère généralement pas que les juristes, par leur activité, pourraient modifier l'avenir et tendre à éliminer les erreurs juridiques les plus diverses, à anticiper et à prévenir les conflits, qui sont générateurs de pertes financières et de malentendus entre les agents économiques. Il en découle que le segment professionnel que nous nous proposons d'étudier n'est pas délimité avec précision. Il nous a fallu construire statistiquement notre objet en même temps que nous cherchions à en connaître les fonctions sociales ${ }^{4}$.

7 La première partie de ce texte est consacrée à la description de la population étudiée. La seconde partie porte sur l'analyse du rôle que cette population tient dans la conduite et le déroulement des affaires internationales concernant l'Égypte.

\section{Caractéristiques de l'échantillon étudié}

8 Dans un rapport de 1988 établi pour le Commissariat général du Plan, Jean-Claude Coulon notait combien il est toujours difficile, à notre époque, de connaître avec précision le nombre des juristes résidant en France, leur spécialisation professionnelle, les formes d'entreprises dans lesquelles ils travaillent ${ }^{5}$.

9 Il en est de même en Égypte. En toute généralité, on peut énoncer que les juristes en rapport, dans ce pays, avec le droit des affaires internationales, et autres que les juges 
(affaires civiles et conseillers d'État) appartiennent soit à des cabinets privés d'avocats, soit à des entreprises publiques. Dans l'un et l'autre cas, ils sont inscrits à l'Ordre des avocats. En effet, cette inscription leur permet d'exercer leur profession et de plaider devant les tribunaux égyptiens. Mais les domaines juridiques dans lesquels ils s'investissent n'étant pas répertoriés, pas plus que leur statut ou leurs lieux d'insertion professionnelle, il en résulte qu'il n'existe pas d'évaluation simple des divers sousgroupes constituant l'ensemble des avocats.

Si l'on ajoute, aux raisons techniques de ce manque d'information, les raisons « de société " précédemment évoquées, on comprend que le chercheur concerné par les prestataires de droit international privé en Égypte doive construire statistiquement l'objet de son observation. Comme on l'a déjà mentionné, le présent texte ne porte que sur les avocats de droit international des affaires fonctionnant eux-mêmes dans des structures privées (cabinets individuels ou sociétés civiles d'avocats). Après avoir brièvement indiqué les sources utilisées au cours de cette première phase, descriptive, on présentera les principaux traits caractérisant la population ainsi constituée.

\section{Sources utilisées}

11 La première source consultée est le Martindale-Hubell Law Directory $(M H L D)^{6}$, répertoire américain qui fournit en effet une liste de law firms ou de law offices égyptiens intervenant dans le domaine international. Comme on le sait, l'inscription d'un cabinet juridique dans ce document n'est pas automatique et dépend du paiement d'un droit d'entrée calculé en fonction des frais d'édition, ainsi que des recommandations du milieu professionnel. Cela dit, les informations dont on dispose avec ce répertoire sont plutôt détaillées puisque, pour chaque law firm, indication est donnée de l'ensemble des avocats qui y travaillent (partenaires principaux, associés, conseillers). Les curriculum vitae sont reproduits pour chaque personne et, en ce qui concerne la firme elle-même, mention est donnée, le plus souvent, de ses principaux clients et marchés. La liste qui en ressort n'est pas exhaustive. Cependant, dans le cas de l'Égypte, pays commercialement très lié aux Etats-Unis, la probabilité est forte de retrouver, dans le $M H L D$, l'essentiel des firmes égyptiennes de droit international des affaires, ou les plus importantes d'entre elles.

12 Outre l'édition du MHLD, il a paru intéressant de demander aux Chambres de commerce implantées en Égypte la liste des cabinets juridiques avec lesquels elles sont en relation ou qu'elles recommandent à leurs ressortissants lorsque ceux-ci les consultent pour réaliser des affaires dans ce pays, ou pour en prospecter les marchés. L'hypothèse sousjacente à cette démarche était double. D'une part, on a supposé que les informations fournies par les Chambres étaient susceptibles de donner une image de la spécialisation (géographique et linguistique) ou au contraire de la non spécialisation des firmes juridiques égyptiennes. D'autre part, les listes ainsi obtenues pouvaient être considérées comme complémentaires de celle du MHLD. Les critères d'inscription sur les listes des Chambres ne sont pas précis et peuvent relever de la compétence, du hasard et du lobbying. Cependant, on peut supposer que les Chambres de commerce s'efforcent d'être à l'écoute du monde local des affaires et réalisent, de ce fait, une certaine sélection dans les listes qu'elles retiennent ${ }^{7}$. 
On trouve en Égypte une information relativement abondante : trois autres sources de documentation ont été utilisées pour constituer l'échantillon de l'enquête. Il s'agit :

- du répertoire des professions annuellement publié par le quotidien Al-Ahrâm (Al-Ahrâm Directory), qui contient une liste d'avocats de droit international (l'édition de 1988 que nous avons consultée en recense une trentaine);

- de la publication réalisée par l'Université américaine du Caire, Cairo, a practical guide. Cet ouvrage de type « grand public » (édition de 1988) répertorie 7 cabinets juridiques ;

- d'informations recueillies auprès de personnes connaissant le milieu professionnel.

$\mathrm{Au}$ total, ces diverses sources d'information ont permis de constituer un échantillon de 26 cabinets. De ces 26 cabinets, nous avons rencontré 1 représentant au moins pour 16 d'entre eux (62\%). Avec les avocats que diverses contraintes ne permettaient pas d'interroger directement, nous avons cherché à établir un premier contact, soit par courrier, soit par téléphone, au Caire et à Alexandrie. On peut donc dire que l'enquête présentée ci-après repose sur la rencontre personnelle des représentants de 16 firmes juridiques (20 entretiens d'une durée moyenne d'une heure), sur des entretiens téléphoniques plus brefs avec les représentants de 6 autres firmes et enfin, sur des contacts épistolaires ou des informations déjà publiées pour les autres (on trouvera en annexe un tableau résumant certains aspects de l'enquête). Nous ne prétendons pas avoir consulté tous les cabinets juridiques égyptiens qualifiés dans l'international. Potentiellement, d'après nos sources, nous étions en mesure, en effet, d'entrer en contact avec 35 à 40 firmes de ce type. Symétriquement, nous ne prétendons pas que les cabinets juridiques égyptiens dont nous avons traité représentent le panel supérieur de cette catégorie professionnelle. Cependant, on peut estimer que l'échantillon comprend à coup sûr les 5 ou 6 gros cabinets égyptiens intervenant dans ce domaine et que les autres, une vingtaine, sont représentatifs de la population restante.

\section{Principaux traits quantitatifs}

Puisqu'il s'agit d'une population, on peut l'étudier selon les critères classiques du nombre, de l'âge, de la taille, de la structure et de la localisation.

\section{Le nombre}

La population des juristes travaillant, à titre privé, dans le droit international des affaires est relativement peu nombreuse. Les 26 cabinets privés de l'échantillon totalisent 263 avocats à temps plein et peut-être une dizaine d'avocats à temps partiel, que nous n'avons pas comptabilisés. La population étudiée représente donc un très petit nombre de l'ensemble des juristes en exercice. Il s'agit d'un groupe restreint, culturellement et statutairement très distinct de la majorité des avocats par sa formation, par ses contacts et ses déplacements internationaux, par ses revenus, voire même par la nature de ces derniers (devises). De cette situation relative peut découler, de la part des autres juristes, un certain ressentiment, avec d'éventuelles conséquences sociales et professionnelles ${ }^{8}$. Mais on peut également constater le contraire. Ces cabinets privés internationaux sont des pôles d'attirance, tant sur le plan intellectuel que sur le plan salarial. 


\section{L'âge} en effet en Égypte une tradition juridique déjà ancienne. L'ordre des avocats y fut fondé en 1912, et l'on sait qu'au XIX ${ }^{\mathrm{e}}$ siècle, les khédives réformateurs consacrèrent au Droit et à la formation juridique une grande part de leur attention ${ }^{10}$. Cependant, ce nombre de cabinets anciens est relativement faible dans l'échantillon, ce qui conduit à évoquer deux phénomènes. générations, lorsque leur structure de base est individuelle ou repose sur une même génération. Les vocations familiales peuvent s'éteindre. On doit noter à ce propos, l'adoption en Égypte, en 1981, d'une loi sur les sociétés civiles d'avocats destinée à lever en partie cette difficulté. En outre, le modèle du cabinet juridique anglo-américain, véritable entreprise fonctionnant selon les critères prioritaires de la rentabilité, tend à s'imposer bien que subsistent, en Égypte, d'importantes limites à son extension. important - est de nature politique. Les avocats égyptiens appartenaient souvent, avant les années 1950, à de vieilles familles terriennes. Celles-ci ont été dépossédées de leurs biens au fur et à mesure que se radicalisait le régime qui mit fin à la monarchie. Après 1952, la rupture progressive avec l'Occident, l'établissement corrélatif de liens avec l'URSS, le conflit israélo-arabe, la nationalisation généralisée d'entreprises et de biens immobiliers ont eu pour effet de réduire considérablement l'activité des cabinets juridiques alors en fonction. L'émigration d'une fraction importante de la population égyptienne active, juive ou autre, appartenant souvent aux catégories intellectuelles et d'affaires, a renforcé le phénomène précédent.

L'une des personnes interrogées explique que le gouvernement égyptien de l'époque n'empêchait pas les avocats d'exercer leur profession à titre privé. Cette personne a donc pu maintenir son cabinet, mais sa clientèle a été réduite des trois-quarts. D'autres facteurs, cependant, ont encore contribué à minorer ce segment professionnel dans la société égyptienne : tout d'abord, le droit donné aux juristes d'entreprises de s'inscrire à l'Ordre des avocats pour qu'en soient modifiées de l'intérieur les orientations politiques et la direction; ensuite, l'influence de l'Union Soviétique sur la pratique juridique des entreprises égyptiennes, désormais publiques mais aussi complètement inexpérimentées.

21 Sur ce dernier point, un avocat note qu'« en 1960, l'État est devenu le grand entrepreneur en Égypte. Les entreprises ont alors ressenti le besoin de disposer de contrats standardisés, ainsi que de leurs avocats et de leurs juristes. La bureaucratie a bien dressé des contrats standards qu'elle a essayé d'imposer, mais ces contrats étaient mal faits, leurs prévisions élémentaires... les entreprises s'y sont brûlé les doigts ". L'influence soviétique n'a pas permis de corriger cette inexpérience. En effet, toujours selon cet avocat, «il y avait un manque de sophistication juridique des deux côtés ». Sur le plan technique, il est semble-t-il résulté de cette situation que lorsque naissaient des conflits de droit dans les relations économiques entre les deux Etats, la tendance naturelle était de les résoudre politiquement et non juridiquement. Ce que confirme un autre avocat, qui appartenait alors au département juridique d'une entreprise publique: "On avait des contrats avec l'Europe de l'Est. C'était une affaire plutôt politique. En cas de conflit, c'était l'ambassadeur, ou même parfois le ministre soviétique concerné qui intervenait ». L'ampleur des travaux faisant

Égypte/Monde arabe, 12-13| 1993 
l'objet des relations entre ces deux pays (Assouan, pétrole, aluminium) ne pouvait qu'encourager ce processus d'envahissement par le politique. Au total, et bien que toutes les relations juridiques n'aient pas été rompues avec l'Occident, notamment dans le domaine bancaire, on peut dire en simplifiant que les années 1955-1974 ont été pour l'Égypte - et pour ce qui concerne le droit international des affaires et les cabinets une période de régression juridique.

On peut voir, dans les éléments que l'on vient de rappeler, une des raisons pour lesquelles les départements juridiques internes des entreprises égyptiennes sont aujourd'hui en retrait par rapport aux exigences du «business » international. À contrario, certains avocats, expatriés pendant ces années pour des raisons politiques, ont effectué, en tant que salariés de cabinets américains ou européens, un apprentissage de formes juridiques modernes dans le domaine examiné. Après le démarrage de la politique de l'infitah, ces avocats sont revenus en Égypte et ont fondé leur cabinet. Le développement des ressources pétrolières à cette époque et leurs effets induits sur l'économie des pays arabes en général ont favorisé ce retour.

Précisément, les années 1974-1980 semblent représenter la grande période de création de cabinets juridiques de droit international dans ce pays. Dans l'échantillon, on en compte au moins 11 (42\%) créés au cours de cet intervalle de temps. Le gouvernement égyptien adopte, en 1974, une loi visant à favoriser l'investissement étranger. Même s'il convient de ne pas surestimer la portée libérale de cette législation, elle indique une rupture par rapport à la phase nassérienne et surtout, sur le plan politique, les EtatsUnis se substituent à l'Union Soviétique. Or l'aide et les prêts américains ouvrent la voie à des compagnies puissantes, solvables, très sensibles aux aspects juridiques de leurs décisions. En outre, la présence de ces compagnies engendre une sorte d'émulation financière chez les autres pays, émulation elle-même génératrice d'achats et de contrats, en sorte que l'activité juridique internationale est, tout à coup, fortement stimulée ${ }^{11}$.

Enfin, la dernière période de création active de cabinets juridiques en Égypte semble avoir débuté en 1987, puisque depuis cette date, l'on en compte six nouveaux dans l'échantillon. Ce phénomène est surprenant dans la mesure où les affaires avec les pays occidentaux ont eu alors tendance à ralentir. Mais il peut s'expliquer par la perspective de la libéralisation de l'économie égyptienne.

\section{La taille}

L'examen de la répartition des cabinets juridiques égyptiens selon le nombre total de leurs avocats à plein temps (taille) montre que la taille modale de l'échantillon est de 8 avocats, sa moyenne arithmétique étant de 10. Ce résultat moyen recouvre l'existence de deux sous-populations. La première est celle des cabinets ( 21 sur 26 , les $4 / 5$ ) dont la taille varie de 1 à 12 avocats. Il semble que, dans cette zone d'importance numérique, il y ait un continuum possible de solutions dans la gestion de ces firmes, en sorte que l'on trouve pratiquement toute la gamme des tailles possibles.

En revanche, le groupe des cabinets ayant environ 20 avocats ou plus (5 sur 26) parait en rupture avec le groupe précédent ${ }^{12}$. 


\section{Les structures par genre et par statut}

\begin{tabular}{|l|l|l|}
\hline-12 firmes & ont à leur tête & 1 non salarié ; \\
\hline-7 firmes & & 2 non salariés; \\
\hline-3 firmes & & 3 non salariés; \\
\hline-4 firmes & & 4 à 8 non salariés. \\
\hline
\end{tabular}
demeure la plus fréquente. Sur les 263 avocats de l'échantillon, 58 (dont au moins 5 professeurs) ont un position dirigeante. Ce qui signifie que chacune de ces 58 personnes gère en moyenne, outre le sien, le travail de 3 à 4 avocats salariés (3,5). L'association, dans le métier juridique, paraît en Égypte (comme ailleurs) un objectif réalisable quoique difficile à atteindre. Un avocat du Caire explique les raisons pour lesquelles il a renoncé à s'associer avec un autre, et a fortiori avec plusieurs: outre les questions déontologiques et le rapport à la clientèle, se posent celles, concrètes, de la gestion du cabinet, de la répartition des charges fixes, de la décision des investissements. Les juristes égyptiens de droit des affaires internationales (mais on observe le même phénomène en Tunisie par exemple, ou au Maroc), résolvent le problème de leur croissance directionnelle par le biais familial, principalement.

La profession d'avocat de droit des affaires internationales est donc, en Égypte, une profession masculine, plutôt individualiste sinon familiale, où les femmes interviennent soit directement, soit par le biais de formes familiales de gestion des cabinets. Pour ce qui concerne les informations sur les gains, les rémunérations et autres données monétaires, nous n'avons pas cherché à les obtenir. Elles relèvent de la confidentialité des affaires. Chacun sait qu'un juriste travaillant dans un cabinet privé d'avocats gagne beaucoup plus que dans la fonction publique égyptienne. Il y a donc une attirance exercée sur le secteur public par le secteur privé, ce qui renforce le dynamisme précédemment évoqué des cabinets juridiques, comparés aux départements correspondants des entreprises. Il s'agit actuellement d'un phénomène général en Égypte.

\section{Les techniques utilisées}

31 Les cabinets d'affaires internationales en Égypte suivent les évolutions techniques indispensables à l'exercice de leur profession. Tous sont équipés d'un système de télex (peu utilisé) et de télécopie (fax). On note, chez la plupart d'entre eux, l'usage croissant $\mathrm{du}$ micro-ordinateur, principalement pour du traitement de textes (courrier, facturation ${ }^{13}$. Ce phénomène, cependant, n'est pas général. On remarque en effet, pour

Égypte/Monde arabe, 12-13 | 1993 
quelques-uns d'entre eux, le refus explicite de l'usage de machines bureautiques modernes, bien que le coût puisse en être considéré comme relativement minime et décroissant. Le micro-ordinateur est alors perçu comme un facteur de modification perturbant du rapport traditionnel à la clientèle. En outre, l'utilisation efficiente de ce matériel (notamment pour enregistrer la législation au jour le jour) suppose l'embauche de personnels éventuellement plus qualifiés, donc plus rémunérés, que les actuels secrétariats ${ }^{14}$.

\section{La localisation urbaine}

32 Comme on peut s'y attendre, ces cabinets sont localisés là où se trouvent les affaires, les infrastructures économiques et les diverses formes du pouvoir d'État. La capitale est leur lieu principal d'implantation. Dans l'échantillon, 2 cabinets seulement ont leur siège social à Alexandrie. Cela dit, en raison de la tradition intellectuelle et commerçante prévalant encore dans cette ville malgré la parenthèse nassérienne, en raison de sa proximité avec la zone franche de Port-Saïd, il y a place, en cet endroit, pour des cabinets importants (dont, par exemple, un des cinq plus gros cabinets égyptiens). Ces cabinets alexandrins ont des antennes au Caire. Leur spécialisation peut être différente de celle des cabinets du Caire (droit maritime, assurances, par exemple).

Pour ce qui concerne les cabinets cairotes, on observe une localisation reflétant certains aspects du développement urbain du Caire. Le centre ville représente le lieu de l'implantation la plus ancienne. Mais la modernisation des immeubles y est coûteuse et difficilement réalisable. C'est pourquoi, si l'on y trouve encore bon nombre de cabinets juridiques importants, d'autres, également importants, ont quitté cet emplacement et se sont installés à Zamalek. Le quartier de Garden City, proche du centre-ville, abrite également plusieurs cabinets mais présente, semble-t-il, des limites de rénovation comparables à celles du centre-ville. La reprise, en 1974, de l'activité juridique internationale se serait accompagnée d'une migration géographique des cabinets cairotes. Leur déplacement vers le nord-ouest s'est poursuivi en direction de Mohandessin-Doqqi, où la nouvelle grande artère (l'avenue de la Ligue arabe ou d'autres avenues proches, en particulier celle du Vingt-six juillet) reçoit plusieurs cabinets juridiques de droit international. Il semble enfin que cette progression se poursuive vers le sud, de façon tournante, en direction du quartier de l'université (Giza). Il s'est donc produit en Égypte, au cours des quinze dernières années, un double mouvement de relocalisation dans la ville et de modernisation immobilière.

Un autre aspect de la localisation urbaine des cabinets égyptiens est celui de leurs extensions, en Égypte même. On observe que deux cabinets cairotes ont ouvert un bureau à Alexandrie et qu'inversement, deux cabinets alexandrins ont une branche au Caire, dont l'un est en outre représenté à Port-Saïd et à Suez.

\section{Les liaisons internationales}

Dernier élément de description des cabinets égyptiens d'affaires internationales : leurs liaisons avec d'autres cabinets occidentaux ou leurs implantations externes. Sous cet angle, l'Égypte apparaît comme un cas limite, intermédiaire de ce que l'on peut observer dans la Péninsule arabique (une quinzaine d'associations avec des cabinets occidentaux, pratiquement tous anglo-saxons), d'une part, et dans les pays du Maghreb 
(relations informelles de correspondance avec les grands cabinets occidentaux), d'autre part ${ }^{15}$.

Nous indiquerons maintenant les principales fonctions socio-économiques des entreprises juridiques égyptiennes travaillant à l'échelon international.

\section{Les firmes égyptiennes de droit des affaires : des médiateurs obligés}

37 Les juristes de droit des affaires internationales sont, de nos jours, les médiateurs obligés des échangés économiques. Si un exportateur ou un investisseur occidental, par exemple, souhaite entrer en rapport avec le marché et les opérateurs égyptiens, la connaissance de la législation et de la pratique juridique relatives à son activité en est une des conditions nécessaires. Il pourra se satisfaire d'une image approximative de l'ampleur du marché et des revenus qui l'alimentent, mais il lui faudra une vue précise du contenu du droit local et de la manière dont il fonctionne. C'est pourquoi, pour des raisons de formation universitaire et professionnelle, pour des raisons de langue, d'expérience du pays et de connaissance concrète des personnes situées aux postes de commande, les avocats égyptiens sont les mieux placés pour rendre ce type de service.

Ces fonctions de médiation sont au nombre de quatre. Elles concernent l'aménagement des rapports avec l'administration économique égyptienne, la connaissance concrète des décideurs, l'adaptation à la mentalité des hommes d'affaires égyptiens, les rapports avec le système judiciaire.

\section{Les rapports avec l'administration économique}

39 L'administration est, en Égypte, un vaste organisme avec lequel un entrepreneur étranger doit, un jour ou l'autre, travailler et composer. En effet l'administration, d'une part, veille à l'application des lois et règlements qu'elle élabore, d'autre part occupe toujours, par l'intermédiaire du secteur public, industriel et commercial, une place majoritaire dans la vie économique du pays.

Or, il semble bien que le fonctionnement de cette administration (du moins ses parties concernées par les relations économiques avec l'extérieur) se caractérise par une certaine lenteur, et parfois une grande part d'arbitraire. Les avocats d'affaires internationales consultés dans le cadre de cette étude en ont fourni de nombreuses illustrations.

L'un d'eux, par exemple, note que

«les hommes d'affaires occidentaux ont l'habitude de travailler dans leur propre environnement. Mais ici, l'approche est différente et le temps n'est pas important. Un client souhaitait faire enregistrer une filiale. Au cabinet, nous avons constitué le dossier en trois semaines. Ce dossier est maintenant sur le bureau du ministre depuis sept mois.. Or on nous a dit, il y a quatre mois, que tout était en règle. Tout ce qu'il nous faut, nous a-t-on assuré, c'est la signature du ministre. Que serait-ce si le dossier n'était pas en règle ! Mais ainsi vont les choses. Il faut être patient... "

Un autre avocat résume dans les termes suivants certains aspects négatifs de son expérience de négociation avec l'administration :

«Ici, en Égypte, un accord n'est pas un accord au sens occidental du terme.

Supposons qu'après avoir bu des litres et des litres de thé, vous vous entendiez avec 
les autorités égyptiennes et que vous aboutissiez enfin à un accord. Vous rentrez chez vous. Vous êtes content. Mais dans $90 \%$ des cas, on vous appellera le lendemain pour vous dire qu'on vous propose d'ajouter ceci et cela. La proposition nouvelle que l'on vous fait aura un contenu différent de ce que vous aviez conclu la veille ».

\section{peut maitriser le nombre de lois et décrets en vigueur. Il souligne, avec un regard} critique, les raisons de cette abondance :

«Un nouveau code commercial a été voté récemment, mais le projet en était terminé il y a 20 ans déjà. Il avait été rédigé par un groupe de juristes éminents, parmi lesquels Mohsen Chafiq. C'est aujourd'hui un homme d'un certain âge et il désespérait de voir ce projet aboutir de son vivant. Sur la base de cet exemple, on pourrait penser que la pratique législative égyptienne est pondérée et que, dans ce pays, on prend le temps de faire les choses. Ce n'est pas du tout cela. Car pendant ces années, il y a eu, dans ce domaine, un déluge de lois et de sous-lois. On publiait un texte et le lendemain, on se rendait compte qu'il y avait une lacune, une faute, une incohérence. On publiait alors un amendement à ce texte. Il est arrivé que dans le même Journal officiel paraissent la loi, son décret d'application et un amendement à la loi. Ce qui signifie que d'une part, le gouvernement est assez interventionniste dans le domaine des affaires, mais que d'autre part, cet activisme législatif ou réglementaire aboutit à des incohérences répétées ".

Quelle que soit l'origine exacte de ce comportement, il s'ensuit qu'un opérateur étranger doit prendre appui sur les compétences juridiques locales ${ }^{16}$.

Certes, le fonctionnement des administrations économiques égyptiennes ne saurait se réduire aux aspects que font apparaître ces citations. Si elles en indiquent la dominante, encore actuelle, il ne faut pas négliger l'existence de tendances contraires. La libéralisation économique devrait réduire et simplifier l'intervention législative de l'État. Mais une telle réorientation prend nécessairement du temps. En attendant, les avocats égyptiens auront à repérer ces évolutions, surtout les plus erratiques, et à trouver les ajustements réglementaires permettant aux parties concernées de se rencontrer.

\section{Les rapports personnels avec les décideurs publics}

Compte tenu de ce qui vient d'être dit concernant la fébrilité parfois excessive de l'administration égyptienne, ou sa lenteur à prendre des décisions ou à fournir les autorisations nécessaires, fussent-elles de nature courante, il est clair que la médiation d'un avocat connaissant personnellement tel ou tel décideur peut aider à l'avancement des dossiers. Dans la mesure où l'intervention économique de l'État est grande, les avocats d'affaires sont conduits à entretenir des rapports parfois étroits avec le milieu politique. L'efficacité globale d'un cabinet juridique, particulièrement dans un pays arabe en voie de développement et à forte intervention publique, est un mélange de savoir technique et d'aptitude au « lobbying ».

Les meilleurs cabinets sont, évidemment, ceux qui allient la qualité du travail et la qualité des relations au niveau du pouvoir politique. Lorsque les deux facteurs n'ont pas un poids égal, il peut se faire que la facilité d'accès aux décideurs soit considérée comme plus importante que l'aptitude technique. Ainsi, une compagnie d'aviation occidentale à laquelle on fait une remarque sur la réputation de son conseil juridique répond que "sans doute, ça n'est pas le meilleur, mais il connaît bien le ministre ». Un avocat 
raconte, à propos d'un de ses collègues égyptiens, aujourd'hui retraité, l'anecdote suivante : "C'était un homme très compétent sur le plan juridique. Mais un jour, quelqu'un m'a dit: "On ne le voit pas souvent". Il négligeait l'aspect "relations publiques" indispensable du travail juridique dans ce pays». La qualité d'" ancien ambassadeur" ou d'" ancien ministre » peut ouvrir des portes. Il n'est pas rare, en Égypte, de trouver des retraités de la magistrature ou de la fonction publique qui, établis à leur compte, cherchent à vendre, comme avocats ou conseillers juridiques, leur aptitude - réelle ou supposée - à accéder à tel ou tel niveau de décision. Il s'agit assurément d'une fonction importante en raison de la personnalisation des relations sociales, observable en Égypte comme dans la plupart des pays méditerranéens.

\section{L'adaptation à la mentalité des hommes d'affaires ou des décideurs égyptiens}

Etant donné leur formation, leur milieu social d'origine ou pour d'autres raisons (par exemple, émigration pendant la période nassérienne) les avocats rencontrés témoignent d'une parfaite connaissance de la culture de leur pays et de celle, au moins, d'un autre pays occidental. Plusieurs de ces avocats ont fait des études supérieures approfondies en France et aux Etats-Unis. Ils ont travaillé dans des cabinets européens ou américains. Certains ont occupé des postes de haute responsabilité technique au sein de la Ligue Arabe ou comme conseillers dans les Etats du Golfe. Plusieurs d'entre eux, hommes ou femmes, sont professeurs à l'Université. Il est possible que ces caractéristiques intellectuelles soient en cours d'évolution mais pour l'instant, elles sont observables chez des avocats d'un certain âge et chez leurs collaborateurs.

Il en résulte que cette catégorie professionnelle connait les comportements des uns et des autres et peut donc contribuer à les ajuster. Une avocate explique, par exemple,

«que les Égyptiens tendent à conclure un contrat avec très peu de détails. Il leur suffit d'un mot et demi pour s'engager. Ils ne commencent à discuter le contrat que s'il y a conflit. Les Occidentaux, au contraire - Américains ou Italiens, puisque ce sont mes principaux clients - veulent tout comprendre à l'avance. Ils veulent connaître toutes les possibilités, tous les arrangements ».

Lorsque cette personne incite la partie égyptienne de sa clientèle à mieux saisir les tenants et aboutissants du contrat qu'elle s'apprête à signer, elle contribue à limiter du moins peut-on le penser - les risques ultérieurs de conflits avec la partie occidentale. De façon complémentaire, la discussion et le contact qui s'établissent entre les deux parties au cours même de cette clarification développent les relations personnelles auxquelles les Égyptiens sont sensibles. De ce rôle médiateur, les exemples sont nombreux. Un avocat égyptien connaissant parfaitement la France dit que bien souvent, lorsque la discussion n'avance plus, les parties égyptienne et française lui demandent son arbitrage, ce qui le met mal à l'aise :

«Le plus grand problème qu'on rencontre - et qu'en tout cas je ressens - est que la manière de penser de chacun est très différente. Du coup, les deux parties se méfient l'une de l'autre. Mon rôle propre devient alors très important, au point que parfois, cela m'effraie. En tant qu'avocat, je ne dois pas influencer la décision, et voilà qu'ils me poussent à être une sorte d'arbitre ».

51 Ce rôle médiateur repose aussi sur le fait que la connaissance linguistique de ces avocats va bien au-delà des rapports de courtoisie. Elle est connaissance de la langue juridique et de la pensée qui l'accompagne. 

nécessairement que l'une des deux parties est plus habituée que l'autre aux affaires modernes. Elles expriment aussi la diversité des comportements humains. Un avocat londonien raconte, par exemple, qu'un importateur égyptien avait fait affaire avec un Anglais, puis n'avait pas payé. Le problème ayant été porté devant le tribunal, l'importateur égyptien expliqua qu'il avait téléphoné à son partenaire britannique dès réception de la marchandise et lui avait dit, sans plus, qu'« il faudrait se voir». Le Britannique acquiesça par politesse à cette proposition sans lui donner suite. Il allait s'ensuivre, d'après l'avocat relatant l'anecdote, divers malentendus. Pour un Égyptien, en effet, il est très impoli d'annoncer brutalement par téléphone à son partenaire commercial que la marchandise ne convient pas. L'importateur avait donc proposé à l'homme d'affaires britannique de le rencontrer. Pour un Européen, en revanche, pour lequel le temps s'identifie à l'argent, la relation personnalisée a perdu sa signification immédiate dans les affaires. Quand l'Égyptien a dit à l'Anglais qu' " il faudrait se voir ", ce dernier n'a pas perçu le moins du monde le sens du message. Quant au juge britannique auquel le litige était soumis, il condamna l'importateur égyptien pour la raison que sa communication téléphonique, sans autre précision, « valait acceptation » de la marchandise.

Ce sont là un ensemble de raisons, mentales et comportementales, pour lesquelles les entrepreneurs occidentaux ont tout intérêt à s'entourer du conseil d'un avocat local. L'avocat d'affaires, s'il a une réelle compétence internationale et connaît bien les cultures dont il assure le face-à-face, n'est pas seulement un technicien du droit. Sans devoir se transformer en sociologues ou en anthropologues, les avocats d'affaires internationales doivent avoir cette sensibilité dont les hommes d'affaires ont le plus grand besoin, à l'étranger, pour agir dans la durée.

Notons que pour faciliter cet accès local à certaines entreprises étrangères moins hardies que les autres, des avocats occidentaux (surtout britanniques) se sont installés au Caire et travaillent en association ou en collaboration avec des cabinets égyptiens ${ }^{17}$. Les hommes d'affaires occidentaux considérés, ou des cabinets juridiques occidentaux, s'adresseraient à ces Britanniques qui serviraient de médiateurs avec le milieu légal et professionnel égyptien. On serait, avec eux, en présence d'un «système à double détente ».

\section{Le contentieux}

55 Il existe une fonction technique pour l'accomplissement de laquelle la présence de l'avocat égyptien est obligatoire, à savoir le contentieux. Les affaires conclues en Égypte ne se traduisent pas inévitablement par des conflits, mais cela peut arriver. En outre, un entrepreneur étranger négligeant les problèmes que peuvent induire les différences culturelles ci-dessus évoquées, court un risque élevé d'avoir à se rendre en justice. Il lui faudra donc un avocat égyptien pour le représenter. Cette obligation se justifie non seulement parce que c'est la loi, mais pour des raisons de fait : la langue du système judiciaire est l'arabe ; le droit civil et commercial égyptien, bien que proche du droit continental européen, s'en distingue sur quelques points essentiels ; la législation, comme on l'a indiqué plus haut, change fréquemment; les comportements des juges, surchargés de procès et secondés par des experts peu rémunérés, doivent être appréciés à leur juste valeur par les avocats.

Égypte/Monde arabe, 12-13| 1993 

soulignent l'extrême longueur de la plupart des procès, même s'il existe quelques chambres rapides. Ils recommandent le plus souvent, lorsque les contrats portent sur de grosses sommes, le recours à l'arbitrage commercial international. Mais dans ce cas aussi, la procédure peut être longue si, dans l'hypothèse où la partie égyptienne est perdante, l'exequatur de la sentence est refusé ou repoussé dans le temps. L'appui d'un avocat local est d'autant plus utile qu'il convient de suivre les procédures d'appel ou d'examiner les refus d'application des sentences arbitrales. Comme on le sait, il existe au Caire, depuis 1978 (opérationnel depuis 1983), un Centre régional d'arbitrage, actuellement dirigé par le Dr. Abu-l-Enein. Compte tenu de la concurrence existant entre la Cour Internationale de la CCI et ce Centre régional, compte tenu aussi de la diversité des soutiens que ces organismes peuvent recevoir (la Coface française recommanderait plutôt l'arbitrage CCI, la Chambre germano-arabe appuierait plutôt le Centre régional du Caire, par exemple), la question doit se poser, pour les avocats égyptiens de droit international des affaires, de savoir comment orienter leur clientèle pour le choix d'un centre d'arbitrage ${ }^{18}$. avocats égyptiens exerçant dans le domaine commercial international : la connaissance pratique de l'administration, l'accès personnalisé au pouvoir, la connaissance de la mentalité et des comportements des agents économiques, l'accès au système judiciaire égyptien en cas de conflit.

La population des avocats d'affaires accomplit le plus souvent l'ensemble des tâches associées à ces fonctions. Les cabinets qu'ils dirigent, law firms ou law offices, conformément à la désignation anglo-saxonne dont ils se qualifient eux-mêmes, sont, pour la plupart, des cabinets de type familial et artisanal effectuant à la fois du conseil juridique et fiscal ainsi que du contentieux, sans spécialisation précise, avec des associés de compétences et d'âges différents selon ces deux secteurs d'activité. Il semble que la partie « contentieux » du travail de ces cabinets se décompose elle-même en deux sous parties : le contentieux international, le contentieux interne. L'existence d'une fonction de contentieux interne est pour ces cabinets une garantie de ressources, et, d'une certaine manière, d'indépendance par rapport aux cabinets occidentaux. Mais elle est, en même temps, révélatrice des limites du marché juridique égyptien de l'international. Plus un cabinet est intégré dans l'activité internationale d'affaires, et moins il pratique de contentieux interne.

L'assiette financière de ces cabinets et le marché qu'ils servent sont actuellement trop réduits pour permettre la mise en place, en Égypte, de «firmes" juridiques à l'américaine. On doit cependant noter, au sein de cet ensemble, l'existence de modalités actives de différenciation et de spécialisation (sur les détails de cette spécialisation, voir l'annexe à cet article). Les plus importantes concernent la taille et la sphère géographique d'activité. En effet on distingue, au sein de la population enquêtée, une sous-population de «gros» cabinets. Par ailleurs, malgré leurs compétences juridiques et pluriculturelles affirmées, tous ces cabinets tendent à se spécialiser selon la «langue juridique ", c'est-à-dire selon la clientèle avec laquelle ils travaillent. À ces spécialisations externes correspondent des formes internes d'organisation, d'autant plus évoluées et sophistiquées que le cabinet est lui-même de grosse taille. Cela dit, le cabinet égyptien type travaillant dans le domaine international est le cabinet bilingue, avec séparation interne du travail entre ceux (les partners) qui assurent le rapport

Égypte/Monde arabe, 12-13 | 1993 
international proprement dit (en langue occidentale) et ceux (les associés, ou salariés du cabinet) qui plaident devant les cours égyptiennes (arabophones). Dans le cas d'un cabinet très impliqué au niveau international, le contentieux tend à n'être que du contentieux d'affaires internationales.

\section{Conclusion}

$60 \mathrm{Au}$ terme de cette présentation des cabinets égyptiens de droit des affaires internationales, étudiés sur la base d'un échantillon large, il paraît possible, en en rappelant les principaux traits, de mieux cerner la différence existant entre eux et leurs homologues européens ou américains.

61 Créés pour la plupart après 1974 , ce sont généralement des cabinets plus petits, et surtout de nature plus familiale. Ils ne sont pas spécialisés : ils tiennent lieu à la fois de conseils juridiques et fiscaux, s'occupent de contentieux externe, voire interne et leurs principaux avocats interviennent parfois comme arbitres. Cela se traduit chez eux par une organisation bipolaire : rapport avec l'étranger, d'une part, rapport avec les cours égyptiennes, d'autre part. Une division de l'usage des langues recoupe cette division professionnelle. Leur équipement technique tend à se moderniser.

62 À quelques exceptions près, ces cabinets sont égyptiens. Le marché juridique de l'Égypte, déjà chargé, paraît trop étroit pour être exploré par de grandes firmes juridiques étrangères, même si certaines y sont représentées (par des partenaires ou en association).

63 Cette caractéristique est importante pour comprendre l'état actuel de cette profession en Égypte. Affaiblie par les conséquences de la politique nassérienne, elle n'a pas bénéficié des potentialités offertes par le marché de la Péninsule arabique ou de la Libye. Il semble que seul Maître Hilmi dirige un cabinet implanté à Riyad ${ }^{19}$. Mais de manière générale, si les juristes égyptiens ont rédigé les constitutions de leurs riches voisins, ils n'ont pas bénéficié du flux de leurs affaires, n'ayant pas le droit d'y plaider ou d'y gérer des cabinets à leur compte ${ }^{20}$. Ces circonstances ont donc cantonné en Égypte les cabinets à vocation internationale, alors que par ailleurs, l'économie de ce pays, majoritairement publique, se montrait très peu utilisatrice de leurs prestations juridiques. De ce fait, ces cabinets n'ont pu constituer aussi rapidement que ceux des pays développés des capacités financières suffisantes. Le secteur public égyptien, fort de ses juristes internes et peut-être insuffisamment attentif aux exigences contemporaines dans le domaine du droit des contrats internationaux, ne les a pas, non plus, poussé vers l'extérieur.

Il résulte de ces forces diverses et complémentaires que les cabinets juridiques égyptiens de droit des affaires internationales fonctionnent aujourd'hui principalement comme institutions d'accueil de sociétés étrangères. La mondialisation et la privatisation accentuées de l'économie égyptienne pourraient les inciter au contraire à sortir de l'Égypte et leur en donner les moyens financiers. 


\section{ANNEXES}

\section{Année de création et nombre d'avocats}

\begin{tabular}{|l|c|c|c|c|}
\hline \multicolumn{1}{|c|}{ Cabinet } & Creation & Partenaires & Assoctts & Total \\
\hline 'Abd al-Nur & 1948 & 1 & 8 & 9 \\
'Abd al-Chahid & 1989 & 1 & 1 & 2 \\
'Abd al-Naga & 1980 & 1 & 10 & 11 \\
Bassim & 1988 & 1 & 7 & 8 \\
Al-Gaddawi, Fu'ad & 1976 & 2 & 6 & 8 \\
Al-Ghatit & - & 1 & 7 & 8 \\
Fox \& Gibbons & 1977 & 1 & 2 & 3 \\
Garana & 1947 & 2 & 10 & 12 \\
Hachim, lbrahim, Tawiiq & 1954 & 5 & 20 & 25 \\
Hasuna, Abu 'Ali & 1987 & 2 & 3 & 5 \\
Hilmi, Hamza & 1980 & 2 & 6 & 8 \\
Husni (Mme) & 1987 & 1 & 0 & 1 \\
Ibrachi, Dermakar & 1955 & 3 & 5 & 8 \\
Kamnel & 1974 & 3 & 17 & 20 \\
Kocheiri, Rachid (Mme) & 1978 & 6 & 14 & 20 \\
Mansur & - & 3 & 6 & 9 \\
Sadiq, Fadil & 1988 & 2 & 5 & 7 \\
Chalakani & 1912 & 8 & 23 & 31 \\
Charqawi & 1980 & 1 & 4 & 5 \\
Charif Sa'd. & 1989 & 1 & 6 & 7 \\
T. G. Consultants & 1975 & 1 & 3 & 4 \\
Yasin & 1984 & 4 & 5 & 9 \\
Yahia 'Abd al-Wahud & 1974 & 1 & 7 & 8 \\
Zeizaa & - & 1 & 4 & 5 \\
Naduri, Nahas & 1978 & 2 & 5 & 7 \\
Yansuni, al-Dib & 1872 & 2 & 21 & 23 \\
\hline total & 26 & 58 & 205 & 263 \\
\hline
\end{tabular}

Nous nous proposons ci-après de décrire quelques traits majeurs des cabinets égyptiens les plus importants par la taille. Nous examinerons ensuite les cabinets juridiques plutôt spécialisés dans le rapport avec des sociétés françaises.

\section{Les cabinets les plus importants}

Ce sont ceux qui rassemblent de 15 à 30 juristes, partenaires et associés. En 1991, on comptait 5 gros cabinets de ce type en Égypte, 4 d'entre eux étant localisés au Caire et 1 à Alexandrie. Leur taille est comparable à la taille moyenne des cabinets de notre échantillon, soit 7 à 8 juristes.

Un des réflexes compréhensibles d'un entrepreneur ignorant tout d'un domaine particulier peut être, lorsqu'il se trouve en pays étranger, de s'adresser aux « gros » en priorité, l'idée étant que si leurs prestations sont éventuellement plus chères que celles de leurs concurrents, la probabilité est aussi plus grande pour qu'elle soit de meilleure qualité. Voici, cabinet par cabinet, quelques caractéristiques essentielles des plus importants d'entre eux. 


\section{1) Zaki Hachem, Muhammad M. Ibrahim, Usama Tawfiq}

Le cabinet du Dr Hachim a été créé en 1954. Il a d'abord dû faire face aux difficultés rencontrées à cette époque par les juristes d'affaires (hostilité du régime de Nasser et faiblesse du marché égyptien). Au début des années 1970, il aurait été le premier à se lancer dans l'activité juridique internationale et à se structurer comme une entreprise.

Ce cabinet, qui a vu passer chez lui et a formé plusieurs des juristes actuellement importants sur la place du Caire, totaliserait 25 lawyers. Il semble travailler principalement avec des compagnies américaines. On y trouve des juristes égyptiens formés à l'ancienne, c'est-à-dire bilingues et souvent trilingues dans la matière juridique. Un entrepreneur français pourra y rencontrer des personnes (le Dr Ibrahim, par exemple) connaissant parfaitement la langue et le droit français. Le Docteur Hachim a lui-même une double formation, française et américaine.

\section{2) Le cabinet des Frères Kamel}

KLO (Kamel Law Office) est sans doute un des cabinets les plus dynamiques du Caire pour ce qui concerne le recrutement. Créé en 1974, ce cabinet compte aujourd'hui 22 juristes (partenaires, conseillers, associés). L'un des « partenaires » de KLO est John Bentley, un juriste américain qui a participé à la préparation des lois de 1974. Une caractéristique importante de KLO semble être d'inscrire sa vocation internationale dans son recrutement. Car si l'on y trouve des personnes qui, tel le Dr Gamil Chehata, ont une connaissance parfaite du droit français, on y trouve aussi des juristes de nationalité étrangère accomplissant des stages, dont certains français comme Me JeanFrançois Rycx, actuellement avocat à Marseille, qui y a exercé son activité pendant plusieurs années. Notons également que KLO est, depuis 1987, en « joint-venture » avec Fox and Gibbons (cabinet londonien spécialisé dans les pays du Golfe, implanté en Égypte depuis 1977).

Quelques entreprises et secteurs clients de KLO stricto sensu: Denison Mines, Caltex, Manufacturers Hanover Trust, Union Bank of Finland, Gillette, Nestlé, Philip Morris, Reuters, Xerox, Elf Aquitaine.

\section{3) Le cabinet de Muhammad Kocheiri et de Samia Rachid}

Ce cabinet, créé en 1978, est le plus petit des cabinets importants de notre recensement (17 juristes). Il s'agit d'un cabinet familial dirigé par deux personnalités liées à l'enseignement supérieur égyptien, Mme Rachid, professeur à l'université du Caire, épouse du Dr Kocheiri, vice-président de l'université Léopold Senghor d'Alexandrie. La caractéristique familiale est fréquente pour ce qui concerne les cabinets égyptiens. Les enfants de ces personnes sont maintenant partners du cabinet.

Le Dr Kocheiri a commencé son apprentissage professionnel aux Etats-Unis, au siège social de Baker et Mac Kenzie (Chicago). Sa spécialité serait l'arbitrage commercial international : il en aurait effectué 46 à ce jour (1991). Il est membre, notamment, du Conseil pour l'arbitrage international (ICCA) ainsi que du Conseil d'arbitrage francoarabe (SEEA). 


\section{4) Le cabinet Chalakani}

C'est incontestablement, depuis son installation à Zamalek, le plus moderne des grands cabinets égyptiens de l'international au Caire. C'est aussi un des plus anciens, puisqu'il fut fondé en 1912 par Me Ali 'Abd al-Fattah Chalakani. Si l'on en juge par l'architecture de ses nouveaux locaux, le cabinet juridique moderne d'aujourd'hui tend à devenir une entreprise intellectuelle de pointe.

Le cabinet Chalakani totaliserait une trentaine de juristes (1991) répartis en trois sousgroupes, prenant chacun en charge, sous la direction des partners, un domaine particulier. L'organisation du travail y est donc plus avancée, et peut-être plus efficace, que dans d'autres cabinets. La clientèle en est surtout - quoique non exclusivement américaine, comme celle du cabinet Hachem, avec, semble-t-il, une dominante bancaire. Depuis 1981, le cabinet Chalakani a un bureau à Alexandrie.

Quelques entreprises et secteurs clients : secteur bancaire (Citibank, Bank of America, Chase Manhattan, Lloyd's Bank, Crédit Suisse, Hong Kong Egypt Bank, National Bank of Pakistan, Paribas, Saoudi Egypt Investment and Finance Company, Joint Arab Investment Corporation). Entreprises industrielles et de construction, hôtels et services.

\section{5) Le cabinet Jean Yansuni et al-Dib}

C'est le plus ancien (120 ans) des cabinets égyptiens d'affaires internationales actuellement en activité. Toujours implanté de manière principale à Alexandrie, ce cabinet de 26 juristes (en 1991) dispose en outre de 3 antennes, une au Caire (3 collaborateurs), une à Port-Saïd ( 3 collaborateurs), une à Suez ( 2 collaborateurs). Il est affilié au cabinet « Hoda 'Abd al-Hadi et Partners », agents de brevet (Le Caire, Alexandrie).

Ce cabinet a une forte compétence en droit maritime. Ses clients sont donc d'abord la quasi-totalité des mutuelles d'assurances maritimes présentes en Égypte, soit notamment les mutuelles britanniques, hollandaises, Scandinaves, russes et chinoises, lesquelles représentent $60 \%$ de l'armement mondial. Dans le domaine bancaire, ce cabinet compte parmi sa clientèle la Citibank, la Lloyds Bank, la Bank of America, ainsi que Gulfstream Resources (Canada), Coca-Cola, Colgate, Shell International, Consolidated Petroleum Company, Warner and Lambert, Squibb Corporation. Les correspondants européens de ce cabinet sont principalement londoniens.

Le trilinguisme arabe-anglais-français est la compétence juridico-linguistique minimale de ces cabinets, mais d'autres langues et droits y sont maitrisés : italien et grec, par exemple, pour le cabinet Yansuni et al-Dib, italien et allemand pour les cabinets Hachim et Kamel. Comme l'indiquent les noms de leurs clients, leur intervention internationale est principalement externe, en ce sens qu'ils reçoivent une clientèle étrangère, mais sont encore peu sollicités par les entreprises égyptiennes pour qu'ils les aident à structurer juridiquement leurs marchés extérieurs. Ce fait est une indication sur l'étroitesse du marché juridique égyptien. 


\section{Les cabinets plutôt tournés vers les entreprises françaises}

Certes, les cabinets que nous venons de décrire fournissent des services à ces entreprises et en comptent dans leur clientèle. Mais ils sont, néanmoins, davantage tournés vers le marché anglo-américain que vers l'Europe continentale. En complément, un certain nombre de cabinets juridiques égyptiens, de taille moyenne ou petite, tendent à se spécialiser dans d'autres secteurs géographiques et linguistiques. Le secteur français est un de ces pôles complémentaires de spécialisation. Bien que ce genre de classement ne soit pas dépourvu d'arbitraire, nous avons relevé 4 cabinets dans ce cas.

\section{1) Sa'd 'Abd al-Nur (centre ville)}

Ce cabinet de 9 juristes travaille principalement, à l'heure actuelle, dans des procès devant le « Tribunal des valeurs », instauré par le président Sadate en 1981. En effet, après le lancement de la politique d'infitah en 1973-1974 et le retour en Égypte d'entreprises que le régime nassérien avait nationalisées au début des années 60 , ce cabinet s'est spécialisé dans les problèmes de « dénationalisation et déséquestration de biens » d'entreprises françaises. Le «Tribunal des valeurs » est l'héritier présent de ces litiges. Sa'd 'Abd al-Nur, ancien élève du collège des Jésuites du Caire, est devenu docteur en droit de l'université de Paris en 1947 et a ouvert son cabinet en décembre 1948. Parmi ses clients, on peut citer Yves Saint-Laurent, Paribas, Crédit Lyonnais, Renault, Elf Aquitaine.

\section{2) A.K. al-Gadawwi et Fu'ad Faruq (Zamalek)}

M. al-Gadawwi est professeur de droit international privé à l'université du Caire. Tout comme son partenaire, Me Faruq, c'est un ancien élève du collège des Jésuites. Leur cabinet, créé en 1976 et regroupant 8 juristes a, comme la plupart des cabinets juridiques de taille moyenne, une double compétence de plaidoirie devant la Cour et de conseil juridique et fiscal. Une des particularités de ce cabinet est de proposer à sa clientèle, si elle le souhaite, non seulement des prestations de nature juridique, mais également des études économiques de marché ou de secteur. Ce cabinet paraît particulièrement sensible à la qualité de sa relation personnelle avec ses clients.

\section{3) A. Hani Garana (centre ville)}

Poursuivant l'entreprise de leur père (Me M.Z. Garana), MM. Hani et Wahid Garana dirigent un cabinet dans lequel travaillent une douzaine de juristes. Il s'agit donc de la tranche supérieure de la première population des firmes juridiques égyptiennes, précédemment examinée. Ce cabinet a été fondé en 1947. Une partie de son activité est dévolue au contentieux, une autre aux affaires internationales proprement dites (conseils juridiques et fiscaux, création de sociétés, « joint-ventures », ainsi que des problèmes de brevets, de marques, etc.). Le tourisme est un de ses secteurs actuels d'intervention. 


\section{4) Yasin M. T. Yasin}

Cet avocat a travaillé pendant vingt ans dans divers cabinets juridiques parisiens, dont il a pu percevoir de façon concrète les nouvelles orientations dans le domaine de l'international à partir des années 1970. Outre sa qualité d'avocat, il mentionne, sur sa carte de visite, celle de " consultant financier ». Cette compétence est révélatrice des changements qui se produisent dans le droit des affaires internationales. Les marchés supposent des financements et il n'existe guère de contrats qui se concluent entre partenaires étrangers sans que ces deux aspects soient liés. Cette jonction professionnelle en une seule personne n'est pas encore très répandue. Son cabinet travaille en correspondance avec divers cabinets parisiens (Joseph Hellal, Coutard et Gevelle).

La liste que l'on vient de donner n'est assurément pas exhaustive. Ces cabinets ne traitent pas exclusivement avec une clientèle française. Ils peuvent s'occuper d'intérêts arabes en Égypte, ou de clients allemands, américains, etc. Réciproquement, d'autres qu'eux travaillent avec des entreprises françaises tout en explorant d'autres créneaux. C'est par exemple le cas du cabinet Samih Sadiq et Fadil, en rapport notamment avec le cabinet de l'avocat parisien Philippe Leboulanger. Il reste cependant que, dans les cabinets de cet ordre, il se produit, sinon une spécialisation, du moins la mise en oeuvre d'une dominante géographique. Nous avons donné des éléments relatifs à la dominante égypto-française. Les cabinets les plus importants, ainsi peut-être que Hilmi et Hamza, travailleraient plutôt dans le domaine égypto-américain. En matière de transactions germano-arabes, le nom du cabinet Ibrachi et Dermarkar ou celui de Me Bassim, conseiller juridique, sont avancés. Les clients actuels de Me Dia F. Husni sont plutôt italiens. On rencontre, évidemment, des cabinets pluralistes de ce point de vue, tels que ceux de MM. 'Abd al-Chahid, Abu-l-Naga, Hasuna et Abu 'Ali, Naduri et Nahas, Yahia 'Abd al-Wadud ou Ze'azaa.

\section{NOTES}

1. Sur cet aspect de la critique contemporaine de la perception des faits économiques par les théories courantes, cf. par exemple KARPIK Lucien, «L'Advocatus Economicus » (in Annales des Mines, mars 1992, p. 7-16).

2. Cf. COMMONS John R., Institutionnal economics, its place in political economy (New-York, The Macmillan Company, 1934, 921 p.). Cf. plus particulièrement p.51-58. COMMONS définit la transaction comme une unité de base de l'activité économique "which correlates law, economics and ethics» (p.58). Les économistes "néo-institutionnalistes" (en particulier Oliver E. WILLIAMSON) ont développé cette impulsion initiale à l'aide du concept de «coût de transaction». Ronald J. GILSON caractérise le juriste comme étant un «transaction cost engineer » (in « Value creation by business lawyers: legal skills and asset pricing » (The Yale Law Journal, vol. 94, n² 2, 1984, p. 239-313).

3. Cette enquête a bénéficié du soutien du CEDEJ et de l'Agence cairote du Crédit Lyonnais, que nous remercions, de même que les avocats du Caire et d'Alexandrie, qui nous ont si courtoisement aidé dans notre travail.

4. Bien que l'Égypte ait eu une activité internationale bien avant le XIXe siècle, c'est depuis une quinzaine d'années seulement que les cabinets égyptiens (quelques-uns) se sont ouverts à l'international, au sens contemporain du terme, et ont adopté les structures américaines 
d'organisation et de partenariat. Il est donc compréhensible que la littérature savante sur les juristes ne se soit pas intéressée aux cabinets d'affaires internationales en particulier, au demeurant très peu nombreux, mais se soit surtout centrée sur les rapports politiques entre les avocats et le pouvoir central. Il ne nous est donc pas possible de prendre appui sur ces travaux. C'est par l'intermédiaire de l'étude des «Cours mixtes " (c'est à dire du droit et des structures publiques), mais non celle des cabinets proprement dits, que s'effectue ordinairement l'approche historique (XIXe siècle) des relations commerciales entre l'Égypte et l'Occident. La thèse de Bernard BOTIVEAU, citée ci-après, reflète cette limite dans son approche de l'histoire du droit égyptien. Dans la mesure, aussi, où le droit s'appliquant aux relations économiques internationales est le même que celui s'appliquant aux affaires intérieures (droit civil, droit commercial), la tendance est de négliger les aspects internationaux de l'application de ce droit, et l'infrastructure institutionnelle qui lui est associée.

5. COULON Jean-Claude, Les professions juridiques de services aux entreprises dans l'Europe de 1992 (Paris, Commissariat Général du Plan, novembre 1988, doc. ronéoté, 289 p.).

6. Le Martindale and Hubell Law Directory est un annuaire édité aux Etats-Unis depuis 1935. Pour l'année 1990, le volume consacré aux cabinets d'affaires et law firms hors USA est le volume 7.

7. Contact fut notamment pris avec 1) l'American Chamber of commerce in Égypt (AMCHAM). De cet organisme, nous avons consulté le «Membership Directory» de 1990-1991. 2) La GermanArab Chamber ol Commerce du Caire. Cette Chambre était placée, en 1991, sous la responsabilité d'un juriste, le Dr P. Goefrich, assisté de Mme Riedel-Knisel. Cette Chambre publie la revue German-Arab Trade. 3) La Chambre italienne de commerce, actuellement peu active. La France, de son côté, ne dispose pas de Chambre de commerce dans ce pays. Le Poste d'Expansion Economique organisme dépendant du ministère des Finances, en tient lieu.

8. Une étude plus étendue montrerait que le champ juridique (au sens de P. BOURDIEU) des affaires internationales en Égypte est l'objet de luttes entre trois catégories d'acteurs spécialisés : les avocats privés (notre enquête), les avocats des entreprises publiques et les juristes d'État (juges et conseillers d'État). Entre ces sous-groupes existe, comme en Syrie, un affrontement professionnel dont l'enjeu ultime est celui de l'ouverture ou de la fermeture de l'Égypte au libéralisme économique international. Pour le moment, et à la différence de ce qui se passe en Syrie, la Cour de Cassation égyptienne a contenu l'ambition d'un certain nombre de juristes d'État de voir affirmer la compétence exclusive du Conseil d'État dans les conflits impliquant des entreprises publiques.

9. L'actuel cabinet « Yansuni \& al-Dib» (Alexandrie) est l'héritier du cabinet « Aicard \& Moss » qui fut fondé à Alexandrie en 1872, par association entre un avocat français de Marseille (À. Aicard) et un solicitor britannique (F. Moss). On observe un phénomène comparable dans les pays du Maghreb, où des cabinets créés pendant la période coloniale ont été ensuite rachetés par des juristes nationaux après l'indépendance. En 1872, l'Égypte était indépendante politiquement. Mais les avocats égyptiens y étaient encore peu nombreux et sans organisation propre (création du barreau en 1912).

10. Cf. BOTIVEAU Bernard, Sharia islamique et droit positif dans le Moyen-Orient contemporain : Égypte et Syrie (Aix-Marseille, Thèse pour le doctorat en Sciences politiques, février 1989, 430 p.).

11. Il semble que le cabinet de maître Zaki Hachim ait été le premier à se lancer dans ce nouveau domaine, bientôt suivi par celui de maître Ali Chalakani, tout en adoptant la formule américaine du partenariat et des associés. On note à ce propos que le cabinet Hachim (aujourd'hui Hachim Ibrahim et Tawfiq) n'est pas exactement la «mère de tous les cabinets » d'Égypte, comme le dirait peut-être un chef d'État célèbre, mais en a néanmoins engendré quelques-uns par scissiparité. C'est ainsi que MM. al-Gathit, Hamza et al-Kocheiri auraient été, à diverses époques, membres associés de ce cabinet avant de voler de leurs propres ailes ou de procéder à d'autres expériences juridiques. Il semble que cette procédure de création par division soit naturelle dans des organismes dont la composante en travail intellectuel est élevée. Maître Abu al-Naga était 
membre associé du cabinet Chalakani et maître Samih Sadlq l'était du cabinet al-Kocheiri, avant de fonder l'un et l'autre leur propre office, le premier en 1980, le second en 1989.

12. Les cinq cabinets égyptiens de droit des affaires internationales les plus importants sont, par taille croissante en 1991, les suivants: Frères Kamel, Kocheiri et Rachld, Yansuni et al-Dib, Hachim/lbrahim/Tawfiq, Chalakani. Cet ordre a pu évoluer depuis le moment de l'enquête. En outre, le classement par nombre d'avocats peut différer de celui que l'on obtiendrait à l'aide du chiffre d'affaires. En 1992, le MHLD indique le classement suivant (nombre de partenaires, associés, conseillers) : Hachim [16], Kocheiri [16], KLO [20], Chalakani [24], Yansuni et al-Dib [25]. Le nombre de juristes mentionnés dans le MHLD peut être différent, cependant, du nombre réel. Dans le présent article, les nombres d'avocats indiqués correspondent aux déclarations, en 1991, des personnes interrogées. Le cabinet « Hilmi et Hamza », souvent cité comme un des «gros » de l'Égypte, appartient à la catégorie des « 8 ». Ce cabinet est différent des autres en raison de son affiliation à « Baker et Mac Kenzie ». Sous l'angle de la taille, il s'agit d'un cabinet moyen.

13. Le cabinet Chalakani parait être, de ce point de vue, le plus moderne, techniquement, de tous les cabinets du Caire, puisqu'il disposait, au moment de l'enquête, de 8 micro-ordinateurs fonctionnant en réseau.

14. Il serait intéressant d'observer aussi les modifications qui se produisent sur le plan des secrétariats et de l'aptitude de cette interface à parler une ou plusieurs langues étrangères.

15. En 1991, le tableau des liaisons repérées était le suivant : Hilmi et Hamza (intégration à Baker et MacKenzie, dont maîtres Helmi et Hamza sont partners; association (joint-venture), depuis 1987, entre «Kamel Law Office» et «Fox and Gibbons ». Fox and Gibbons est une petite multinationale juridique britannique (environ 45 avocats en 1992) très implantée au MoyenOrient; liaison très suivie entre le cabinet «M. Z. Garana» et le cabinet parisien «Errera, Ménard, Monteran », ce dernier étant lui-même, semble-t-il, proche de "Arthur Andersen »; représentation de «Sidley \& Austin » par le cabinet cairote «Charif Sa'd ». On sait que « Sidley \& Austin» fut implanté au Caire entre 1983 et 1987, puis ferma sa branche; participation du cabinet « Naduri, Nahas » à l'Association « Interlaw ». On trouve également la présence au Caire, depuis la guerre du Golfe (1990-1991), du cabinet multinational 'Donovan \& Associés'. Donovan serait présent au Koweit et en Égypte par l'intermédiaire du cabinet britannique Alsop Wilkinson (environ 200 avocats et 5 branches). Le bureau koweïtien de ce dernier se serait replié au Caire au moment de la crise du Golfe de 1990-1991. Après la phase militaire de la crise, Alsop serait retourné à Koweit City tout en conservant son antenne cairote. C'est alors, en 1991, qu'il se serait associé à Donovan. À ces liaisons internationales connues (il en existe d'autres, informelles ou discrètes), s'ajoute en 1991 la présence, à Riyad (Arabie Saoudite), du cabinet Hilmi et celle, à Locarno (Suisse), du cabinet al-Ghatit, maitre al-Ghatit ayant exercé dans le département juridique d'IBM pendant plusieurs années. Enfin, on peut mentionner la présence au Caire de cabinets étrangers (exemple de T.G. consultants) agissant à la manière des solicitors britanniques et susceptibles de fournir aux compagnies étrangères une première base d'accueil.

16. Dans une autre enquête, que nous, avions réalisée au Caire en 1990 ( Les entreprises françaises et l'économie égyptienne en 1990 : le rôle des services ", doc. ronéoté, Université de Lille 1, 118 p.), la volatilité de la réglementation égyptienne, dans le domaine notamment de la tarification douanière, était apparue comme une gêne pour les opérations d'investissement. Pour un résumé de cette enquête, cf. DELAUNAY Jean-Claude, "Entreprises égyptiennes et risque égyptien, étude de psychologie économique» (Égypte/Monde Arabe, nº 9, 1er trimestre 1992, p. 47-66).

17. Ainsi en trouve-t-on dans quelques grands cabinets. Les « Frères Kamel », par exemple, sont ceux qui ont le plus poussé la qualification de leur cabinet en ce sens puisqu'ils y rassemblent un associé américain, des stagiaires européens et une association en joint-venture avec le cabinet londonien « Fox and Gibbons ».

18. Un second Centre d'arbitrage aurait été ouvert à Alexandrie en 1992. 
19. Un remarque cependant: dans l'édition 1992 du $M H L D$, la représentation de «Baker \& Mac Kenzie » est assurée, à Riyad, par le cabinet saoudien « Dr. Mosa al-Aibar Légal Advisors ». Il est possible que le Dr. Milmi, partenaire de B \& MK, copartenaire, au Caire, du Dr Hamza (également partenaire de B \& MK) et qui exerçait aussi à Riyad avant 1991, ait dû laisser la place à un juriste saoudien.

20. En 1991, un juriste égyptien déclare qu'au Koweit, par exemple, un avocat égyptien n'a le droit de plaider que deux fois par an devant la Cour de ce pays.

INDEX

Mots-clés : cabinets d'avocats, droit des affaires internationales

\section{AUTEUR}

\section{JEAN-CLAUDE DELAUNAY}

Laboratoire LAST-CLERSE, Université de Lille $1 \mathrm{fr}$ 